\title{
Editorial
}

\section{Family reunion of nuclear hormone receptors: structures, diseases, and drug discovery}

\author{
$\mathrm{H}$ Eric $\mathrm{XU}^{1,2, *}$ \\ ${ }^{1}$ VARI-SIMM Center, Center for Structure and Function of Drug Targets, State Key Laboratory of Drug Research, Shanghai Institute of \\ Materia Medica, Chinese Academy of Sciences, Shanghai 201203, China; ${ }^{2}$ Laboratory of Structural Sciences, Van Andel Research \\ Institute, Grand Rapids, MI 49503, USA
}

Acta Pharmacologica Sinica (2015) 36: 1-2; doi: 10.1038/aps.2014.140

Hormones comprise one of the most effective classes of messengers that transmit cell to cell communication in eukaryotic systems. Cells receive messages of hormones through protein receptors, and then translate the hormone signals into concrete action of physiological responses. Homeostasis of hormone systems is essential for human health, and aberrant regulation of hormone signaling has been associated with many diseases, including cancer, diabetes, and inflammation diseases. As such, targeting hormone signaling pathways has become a major venue of pharmacological intervention of many health abnormalities. There are two major classes of hormone receptors: G-protein coupled receptors and nuclear hormone receptors, both of which consist of the top two classes of the most successful drug targets. Two years ago, we published a special issue on structures, biology and drug discovery of G-protein coupled receptors, which has been well received by drug discovery research communities. This year marked the 30th anniversary of the cloning of the first member of the nuclear receptor family and 25th year of the discovery of orphan nuclear receptors, for which no cognate ligands were known at the time of their cloning. Since then we have witnessed a tremendous expansion of our knowledge of this family of receptors. In this special issue, we collected eleven exciting review articles, highlighting our current understanding of structure, function, disease relevance, and drug discovery of nuclear receptors.

Hormones androgen and estrogen have long been known to play key roles in development, growth, and homeostasis of reproductive systems and their dysregulation is the major cause of prostate cancer and breast cancer. The first article by Tan and coworkers will provide comprehensive review on structures, diseases relevance and drug discovery of androgen

\footnotetext{
* To whom correspondence should be addressed.

E-mail eric.xu@vai.org
}

receptor, the oncogenic driver of prostate cancer ${ }^{[1]}$. The second review article by Ikeda and coworkers covers the roles of estrogen-responsive genes in breast cancer ${ }^{[2]}$. Beside androgen receptor and estrogen receptor, the two of classical steroid hormone receptors in cancers, orphan nuclear receptors COUPTFs also known to play important role in tumorigenesis, and they are respectively reviewed by $\mathrm{Xu}$ and coworkers ${ }^{[3]}$. In addition, the roles of bile acid receptor FXR in liver cancer will be reviewed by Huang and coworkers ${ }^{[4]}$.

Nuclear hormone receptors are also well known for their central roles in metabolic regulation. Classic examples are peroxisome proliferator activated receptors (PPARs) and receptors for glucocorticoids and thyroid hormone, for which roles have been well reviewed in the literatures. In this special issue, we will focus on the emerging roles of orphan nuclear receptors in metabolism. Jiao and coworkers review the regulatory role of FXR in homeostasis of hepatic triglyceride ${ }^{[5]}$. Audet-wash and Giguère review the roles of estrogen-related receptor $a$ and $\gamma$ in metabolic control and related diseases ${ }^{[6]}$. Yan and Xie review the role of constitutive androstane receptor in energy metabolism ${ }^{[7]}$. The subfamily of ROR orphan nuclear receptors have emerged as important regulators of metabolism and immune system. The structures, biology and related drug discovery targeting RORs have been intensively studied by academic and industrial groups, and are viewed by Zhang and coworkers ${ }^{[8]}$. Together, these articles provide an overall view on the converging roles of orphan nuclear receptors in metabolic regulation and their potential as drug targets for metabolic diseases.

In addition to endogenous hormones, various environmental compounds can target members of the nuclear receptor family and cause physiological consequences such as disruption of endocrine regulation. William Bourguet's group provides a comprehensive review on structural aspects of nuclear receptors as targets of environmental compounds ${ }^{[9]}$. Nuclear 
receptors are known to have canonical functions as DNAbinding and ligand regulated transcription factors. In addition to their functions in transcription control, nuclear receptors are also involved in nongenomic actions of cell response to hormones and they are also targeted by regulation of signaling from cell surface receptors. Zhang and coworkers provide a detail review of regulation of nongenomic actions of retinoid $X$ receptor- $\alpha$ by targeting the coregulator-binding sites ${ }^{[10]}$. Li and coworkers review the signaling cascade from cell surface receptors to nuclear receptors in the pathways of bile acid metabolism ${ }^{[11]}$.

The root of nuclear receptors started with studying the classical endocrine hormones such as steroid hormones and thyroid hormone, which are the keystone of the modern pharmacology. The revolution of molecular biology has given the birth of the nuclear receptor family and established their roles in hormone recognition and signaling transduction. Following the recent technological advances in system biology, high-through-put sequencing, proteomics, and RNA biology, the field of GPCRs is posited for a new phase of exciting progresses. With a rich history of drug discovery and deep tradition of cutting edge science, nuclear receptors will continue to be an exciting field of innovative research and drug discovery for the 21st century. We hope that the collection of these eleven reviews will provide distinct insight into this new era of nuclear receptor research and drug discovery.

\section{References}

1 Tan ME, Li J, Xu HE, Melcher K, Yong EL. Androgen receptor: structure, role in prostate cancer and drug discovery. Acta Pharmacol Sin 2015; 36: 3-23.

2 Ikeda K, Horie-Inoue K, Inoue S. Identification of estrogen-responsive genes based on the DNA binding properties of estrogen receptors using high-throughput sequencing technology. Acta Pharmacol Sin 2015; 36: 24-31.

3 Xu M, Qin J, Tsai SY, Tsai MJ. The role of the orphan nuclear receptor COUP-TFII in tumorigenesis. Acta Pharmacol Sin 2015; 36: 32-6.

4 Huang XF, Zhao WY, Huang WD. FXR and liver carcinogenesis. Acta Pharmacol Sin 2015; 36: 37-43.

5 Jiao Y, Lu Y, Li XY. Farnesoid X receptor: a master regulator of hepatic triglyceride and glucose homeostasis. Acta Pharmacol Sin 2015; 36: 44-50.

6 Audet-Walsh É, Giguère V. The multiple universes of estrogen-related receptor $\alpha$ and $y$ in metabolic control and related diseases. Acta Pharmacol Sin 2015; 36: 51-61.

7 Yan J, Chen B, Lu J, Xie W. Deciphering the roles of the constitutive androstane receptor in energy metabolism. Acta Pharmacol Sin 2015; 36: 62-70.

8 Zhang Y, Luo XY, Wu DH, Xu Y. ROR nuclear receptors: structures, related diseases and drug discovery. Acta Pharmacol Sin 2015; 36 : 71-87.

9 Delfosse V, le Maire A, Balaguer P, Bourguet W. A structural perspective on nuclear receptors as targets of environmental compounds. Acta Pharmacol Sin 2015; 36: 88-101.

10 Zhang XK, Su Y, Chen L, Chen F, Liu J, Zhou H. Regulation of the nongenomic actions of retinoid $X$ receptor- $\alpha$ by targeting the coregulator-binding sites. Acta Pharmacol Sin 2015; 36: 102-12.

$11 \mathrm{Li} \mathrm{S}$, Ni A, Feng GS. Bridging cell surface receptor with nuclear receptors in control of bile acid homeostasis. Acta Pharmacol Sin 2015; 36: 113-8. 